Check for updates

Cite this: RSC Adv., 2018, 8, 22853

\title{
The effect of surface immobilized NBD peptide on osteoclastogenesis of rough titanium plates in vitro and osseointegration of rough titanium implants in ovariectomized rats in vivo
}

\author{
Yu Wang, (D) a Chen Zhang, ${ }^{\mathrm{b}}$ Weijian Xu, ${ }^{\mathrm{b}}$ Baixiang Wang, ${ }^{\text {a }}$ Yanhua Lan, ${ }^{\mathrm{b}}$ Mengfei Yu, ${ }^{\mathrm{a}}$ \\ Pinger Wang ${ }^{c}$ and Zhijian Xie*d
}

Successful osseointegration in dental implants depends on balanced activation of osteoclasts and osteoblasts. Osteoporosis up-regulates osteoclast activity, so it is desirable to find effective interventions to inhibit osteoclastogenesis and enhance the osseointegration of implants under these conditions. It has been reported that the NF- $\mathrm{B}$ essential modulator (NEMO)-binding domain (NBD) peptide can prevent osteoclast formation and bone resorption. In this study, we conjugated NBD peptide onto the surface of rough pure titanium ( $\mathrm{Ti}$ ) using the layer by layer technique. We analyzed the surface characteristics and determined the successful NBD integration by the presence of trivial granular structures, increased S elements and hydrophilia. Importantly, we first reported that Ti surfaceconjugated NBD peptide retained its inhibitory effects on osteoclastogenesis by reducing osteoclast sealing zone formation and function. These effects were mediated by a reduction in NFATc1 expression, which in turn regulated integrin $\alpha v \beta_{3}$ and MMP9 by targeting the P65 signaling pathway. In vivo TRAP staining suggested NBD-coating decreased osteoclast formation with less pseudopodia. Micro-CT and histomorphometric analysis demonstrated that NBD-coating enhanced pronounced osseointegration in vivo in ovariectomized rats. This study holds great promise for in vivo use of immobilized NBD peptide and offers an effective therapeutic approach to select more suitable Ti-implant surface modifications for

improving implant osseointegration in osteoporotic patients.

Received 11th April 2018

Accepted 14th June 2018

DOI: $10.1039 / \mathrm{c} 8 \mathrm{ra03116a}$

rsc.li/rsc-advances

\section{Introduction}

Osseointegration, the direct structural and functional connection between living bone and the surface of an implant, is accomplished by bone remodeling around the implant site. ${ }^{1}$ Successful bone remodeling in dental implants depends on the balanced activation of osteoclasts, which resorb the underlying bone tissue, followed by subsequent activation of osteoblasts, which are responsible for formation of new bone tissue in the peri-implant region. ${ }^{2-4}$ Under osteoporotic conditions, estrogen deficiency up-regulates osteoclastic activity, and this osteolysis phenomenon is further intensified. ${ }^{5}$ Thereafter, it is desirable to find useful interventions to promote the initial stability and long-term survival of implants, not only for the normal patients

${ }^{a}$ Department of Implantology, Affiliated Hospital of Stomatology, Medical College, Zhejiang University, Hangzhou, P. R. China

${ }^{b}$ Department of Oral Medicine, Affiliated Hospital of Stomatology, Medical College, Zhejiang University, Hangzhou, P. R. China

'Zhejiang Chinese Medical University, Hangzhou, P. R. China

${ }^{d}$ Department of Oral and Maxillofacial Surgery, Affiliated Hospital of Stomatology, Medical College, Zhejiang University, Hangzhou, P. R. China. E-mail: xzj66@zju. edu.cn but also for the patients suffering from osteoporosis. While the effects of the surface properties or protein modifications of titanium (Ti) implants on osteoblast proliferation and differentiation have been well documented, ${ }^{6,7}$ little is known about their impact on osteoclast proliferation, formation or function. Bone resorption around implants can lead to implant loosening and micromotion problems and is a major cause of failure of dental implants. Therefore, understanding the factors that promote or inhibit osteoclast activity is important for the design of implants.

Osteoclasts derive from the monocyte/macrophage lineage and are the exclusive cells of bone resorption. ${ }^{8-10}$ Bone resorption depends on the interaction of the osteoclast receptor, receptor activator of NF- $\kappa \mathrm{B}$ (RANK) with its ligand, receptor activator of NF- $\kappa \mathrm{B}$ ligand (RANKL) which, together with M-CSF, regulates the activation and differentiation of pre-osteoclasts. ${ }^{11,12}$ RANKL binding activates NF- $\kappa B$ and c-Fos which then induce NFATc1, a master transcription factor for the terminal differentiation of osteoclasts. ${ }^{13}$ NFATc1 promotes the differentiation of osteoclast precursors into mature osteoclasts by inducing the expression of key target genes such as DC-STAMP, cathepsin $\mathrm{K}$, integrin $\alpha v \beta_{3}$ and MMP9. ${ }^{14-17}$ Integrin $\alpha v \beta_{3}$ is 
expressed on osteoclasts and has been reported to be involved in the attachment of osteoclasts to bone. Blocking experiments have shown that the $\alpha v \beta_{3}$ integrin functions as a major adhesion receptor in osteoclasts and is crucial for cell attachment and spreading. ${ }^{18,19}$ Activated mature osteoclasts are polarized and form specialized adhesive structures termed podosomes which essentially mature into the sealing zone (SZ) ${ }^{20,21}$ and bind tightly to the bone surface. The SZ contains specialized ruffled border (RB) membrane domains that maintain a low pH in the SZ by releasing protons via a vacuolar H-ATPase, dissolving the mineral phase which is mainly composed of hydroxylapatite. ${ }^{22}$ In addition, proteolytic enzymes responsible for the cleavage and degradation of organic bone matrix, such as matrix metalloproteinases (MMPs) and cathepsin K, are released into the SZ through the RB. Activated osteoclasts express the enzyme TRAP, which is an important marker molecule for osteoclast activation. ${ }^{23}$

A crucial target of RANKL signaling is the activation of nuclear factor kappa B (NF- $\kappa \mathrm{B}) .{ }^{24} \mathrm{NF}-\kappa \mathrm{B}$ is sequestered in the cytoplasm and upon activation translocates into the nucleus where it binds to $\kappa \mathrm{B}$ promoter sequences and initiates transcription. The NF- $\kappa$ B family/complex consists of NF- $\kappa$ B1 (p105 and p50), NF-кB2 (p100 and p52), Rel (c-Rel), RelA (p65) and RelB. ${ }^{25,26}$ Among these members, p65 plays a particularly important role in osteoclast activation. ${ }^{24}$ The $\mathrm{I} \kappa \mathrm{B}$ family of proteins consists of seven known mammalian members, I $\mathrm{B} \alpha$, $\mathrm{I} \kappa \mathrm{B} \beta, \mathrm{I} \kappa \mathrm{B} \varepsilon, \mathrm{I} \kappa \mathrm{Bc}, \mathrm{Bcl}-3$ and the precursor Rel-proteins, p100 and p105, of which $\mathrm{I} \kappa \mathrm{B} \alpha$ has been the most studied. ${ }^{27}$ In resting osteoclast precursors, p65:p50 heterodimers are bound to I $\kappa$ Bs and retained in the cytoplasm. Upon activation, $\mathrm{I} \kappa \mathrm{B} \alpha$ is rapidly phosphorylated and degraded by the proteasome, thereby freeing p65 which can then enter the nucleus, bind to DNA and activate the transcription of target genes. Phosphorylation of I $\kappa \mathrm{B}$ proteins, mediated primarily by the I $\mathrm{B}$ kinases (IKK) IKK $\alpha$, IKK $\beta$ and $I K K \gamma,{ }^{28}$ is thus crucial for NF- $\mathrm{BB}$ activation in osteoclast formation. The serine kinases IKK $\alpha$ and IKK $\beta$ target serines 32 and 36 of $\mathrm{I} \kappa \mathrm{B} \alpha$ while IKK $\gamma$, also known as NF- $\kappa \mathrm{B}$ essential modulator (NEMO), regulates the other two IKKs. ${ }^{29}$ NEMO interacts with a carboxy-terminal sequence in IKK $\alpha$ and IKK $\beta$ termed the NEMO-binding domain (NBD). ${ }^{30}$ Desai et al. found that a short cell-permeable peptide spanning the IKK NBD reduced inflammatory responses in animal models of inflammation. ${ }^{31}$ Importantly, this NBD peptide disrupted the association of NEMO with the IKKs, blocked NF- $\kappa \mathrm{B}$ activation and prevented osteoclastogenesis and bone resorption in animal models. ${ }^{32,33}$ Therefore, an NBD peptide that selectively inhibits NF- $\kappa$ B activation and bone loss by targeting the NBDNEMO interaction while sparing the protective functions of basal NF- $\kappa$ B activity promises to have great therapeutic value and likely fewer undesired side effects. ${ }^{34}$

An ideal implant would actively promote peri-implant bone healing to support the formation of strong, mature bone at the bone-implant interface necessary for mechanical integration and long-term stability. A promising approach to improve dental implants is to coat the implant surface with bioactive compounds that support bone healing, and several modification methods have been developed that enhance peri-implant bone integration. ${ }^{35}$ Surface coating also allows for the addition of organic biomolecules, and the application of therapeutic coatings has become a rapidly growing research area. ${ }^{36}$ Layer by layer (LBL) self-assembly is a relatively novel and easy technique for surface coating that utilizes static or hydrogen bond interactions between macromolecules and creates organic-coated compounds that are more stable during sterilization and storage. ${ }^{37}$ Therefore, we applied the LBL assembly technique as a multilayer build-up method based on electrostatic interactions to synthesize NBD peptide-coated titanium plates. We selected the natural polysaccharides chitosan (CS) and hyaluronic acid (HA) as the building components of NBD films. ${ }^{38,39} \mathrm{CS}$ is a natural, reactive muco-adhesive that is positively charged under acidic conditions while HA is negatively charged. Both CS and HA are weak polyelectrolytes that are nontoxic, biodegradable and highly biocompatible, and they have to date been tested in various fields including pharmaceutical applications, tissue engineering and drug delivery.

In conclusion, we hypothesized that an NBD peptide conjugated to a Ti implant could improve the balance between bone formation and bone resorption at the implant site and thus present a potential new therapeutic approach to prevent bone loss around dental implants. The goals of this study were to determine whether NBD coating could inhibit osteoclastogenesis on Ti plates and whether an immobilized NBD peptide could synergistically enhance osseointegration around implants in osteoporotic model.

\section{Experimental section}

\section{Preparation of Ti plates and implants}

Pure Ti plates (Grade 4, $10 \times 10 \times 1 \mathrm{~mm}$, Guangci Medical Appliance Company, Zhejiang, China) were polished with wetgrinding silicon carbide (SiC) abrasive paper of various grit sizes (from \#320 to \#1600) during water-cooling and then sandblasted, rinsed with acetone, 75\% alcohol and distilled water for $15 \mathrm{~min}$ each in an ultrasonic cleaner and dried under nitrogen flow. ${ }^{40}$ Subsequently, the Ti plates were treated with a solution containing $\mathrm{HF}$ and $\mathrm{HNO}_{3}$ followed by a solution containing $\mathrm{HCl}$ and $\mathrm{H}_{2} \mathrm{SO}_{4} \cdot{ }^{41}$ Roughed $\mathrm{Ti}$ implants with a diameter of $2 \mathrm{~mm}$ and height of $6 \mathrm{~mm}$ were also obtained from Guangci Medical Appliance Company. These implants were treated as described above. All plates were sterilized by ultraviolet (UV) irradiation.

\section{Preparation of glass slides}

Glass slides $(1 \times 1 \mathrm{~cm}$, Shenyan, Jiangsu, China) were cleaned by sonication in pure water, ethanol and acetone for $15 \mathrm{~min}$, respectively, and then dried under nitrogen flow. The slides were immersed in piranha solution (a mixture of $\mathrm{H}_{2} \mathrm{SO}_{4}$ and $\mathrm{H}_{2} \mathrm{O}_{2}$ at a ratio of $7: 3$ ) at $60{ }^{\circ} \mathrm{C}$ for $2 \mathrm{~h}$, thoroughly rinsed with deionized water and dried using a nitrogen blower. ${ }^{42}$

\section{Fabrication of biological multilayer structure}

CS was purchased from Yunzhou Biochemistry, Qingdao, China and dissolved in $0.2 \%$ acetic acid $\left(5 \mathrm{mg} \mathrm{ml}^{-1}\right)$. HA was 
purchased from Sigma Chemical Co., MO, USA and dissolved in distilled water $\left(0.5 \mathrm{mg} \mathrm{ml}^{-1}\right)$. Functional cell-permeable wildtype NBD peptide (DRQIKIWFQNRRMKWKK-TALDWSWLQTE) was synthesized from lyophilized powder (GL Biochem Ltd, Shanghai, China) as previously described ${ }^{43,44}$ and dissolved in distilled water $\left(1 \mathrm{mg} \mathrm{ml}^{-1}\right)$. The CS, HA and NBD solutions were used sequentially for the LBL technique.

Ti plates and implants were divided into an untreated (control), a CS/HA fabricated and a CS/HA/NBD fabricated group, and all LBL steps proceeded at room temperature. Briefly, Ti plates and implants were immersed in CS for $20 \mathrm{~min}$ to form a layer with a stable positive charge, which initiated LBL assembly process, and were then dipped in HA for 5 minutes, which allowed HA to adhere to the surface electrostatically. For the NBD fabricated group, plates were then submerged in NBD solution for another 5 min. Finally, all Ti plates and implants were dried overnight before use. ${ }^{45}$

\section{Analysis of surface characteristics}

The microtopographic properties of the substrates were assessed by field emission scanning electron microscopy (SEM; SU8010FE-SEM, HITACHI, Tokyo, Japan) and atomic force microscopy (AFM; SPI3800N, Seiko Instruments Inc-SII, Japan). For AFM, glass slides prepared as described above were analyzed since it is difficult to observe surface characteristics on Ti plates. AFM images were recorded in tapping mode at 20$25{ }^{\circ} \mathrm{C}$ in air with a Seiko SPI3800N station (Seiko Instrument Inc.), using silicon tips (NSG10, NT-MDT) with a resonance frequency of approximately $300 \mathrm{kHz}$.

Surface roughness was measured using a portable surface roughness instrument (Surftest, SJ-210; Mitutoyo, Kanagawa, Japan). ${ }^{46}$ The contact angles of the untreated and fabricated $\mathrm{Ti}$ plates were measured with a dynamic contact angle system (SL200B, Solon Tech. Inc. Ltd. Shanghai, China). Ultrapure water was used as the wetting agent at room temperature. Surface chemistry was analyzed by X-ray photoelectron spectroscopy (XPS) measurements on an AXIS ULTRADLD spectrometer (VG ESCALAB MARK II) with a monochromatized Al K-alpha X-ray source operating at $15 \mathrm{kV}$ and $8 \mathrm{~mA}$. The final value reported for each sample is the mean of 3 independent replicates.

\section{Biological layer degradation and NBD release}

NBD-coated Ti plates (CS/HA/NBD) were immersed in $1 \mathrm{ml}$ DMSO at $37{ }^{\circ} \mathrm{C}$. At the indicated timepoints, aliquots of the incubation solution $(500 \mu \mathrm{l})$ were collected and substituted with equal amounts of fresh DMSO. These harvested samples were then stored at $-20{ }^{\circ} \mathrm{C}$. The amount of released NBD was measured using ultraviolet-visible spectroscopy (UV/Vis) by measuring the absorption peak of the NBD at a wavelength of $280 \mathrm{~nm}^{31,32}$ The optical density (OD) of each group was calculated and is representative of the mean of 3 independent experiments.

\section{In vitro assessment of multinucleated cell formation}

RAW 264.7 cells (ATCC; TIB-71, Cell Bank of Chinese Academy of Sciences, China) were cultured in Dulbecco's Modified Eagle
Medium (DMEM; Gibco, Invitrogen Corp., USA) supplemented with $10 \%$ fetal bovine serum (FBS), $100 \mathrm{IU} \mathrm{ml}^{-1}$ penicillin and $100 \mu \mathrm{g} \mathrm{ml}{ }^{-1}$ streptomycin at $37^{\circ} \mathrm{C}$ in $5 \% \mathrm{CO}_{2}$. The media was refreshed every 2 days. Before use, a $20 \mathrm{mM}$ NBD peptide stock solution was prepared in DMSO and diluted to the final concentration in media. To determine the inhibitory role of NBD on osteoclast formation, RAW 264.7 cells were stimulated with soluble recombinant mouse RANKL (R\&D System, Minneapolis, MN, USA $)^{47}$ at $100 \mathrm{ng} \mathrm{ml}^{-1}$ in the presence or absence of NBD peptide $(20 \mu \mathrm{M})^{43}$ in $\alpha$-Minimum Essential Medium Eagle ( $\alpha$-MEM; Gibco, Invitrogen Corp., USA). After 4 days, cells were fixed with $4 \%$ formaldehyde and immunostained with fluorescein isothiocyanate-labeled phalloidin (F-actin) and 4'-6diamidino-2-phenylindole (DAPI) (Sigma-Aldrich, St. Louis, MO, USA). Cells were then evaluated and images were acquired using a Nikon 90i fluorescent microscope (Nikon, Melville, NY, USA). Osteoclasts were also stained for TRAP using a kit by following the manufacturer's instructions (Sigma-Aldrich, St. Louis, MO, USA). The TRAP ${ }^{+}$cells were counted under light microscopy (Nikon, Melville, NY, USA). Multinucleated cells containing more than three nuclei were counted as osteoclasts. Cell morphology was also characterized by SEM.

RAW 264.7 cells were cultured on sterilized untreated, CS/HA or CS/HA/NBD fabricated Ti plates as described above and then stimulated with RANKL ( $100 \mathrm{ng} \mathrm{ml}^{-1}$ ) in $\alpha$-MEM medium. After 4 days, cells were fixed and processed as described above. Multinucleated cells containing more than three nuclei were counted as osteoclasts. Cell morphology was also characterized by SEM.

\section{Cell proliferation assay}

RAW 264.7 cells with or without RANKL (100 ng ml ${ }^{-1}$ ) stimulation were seeded respectively in untreated $\mathrm{CS} / \mathrm{HA}$ or $\mathrm{CS} / \mathrm{HA} /$ NBD fabricated Ti plates at seeding cell density of $1 \times 10^{4}$ cells per well, and cultured for 1, 3 and 5 days, and then cell proliferation was assessed using a cell counting kit-8 (CCK-8, Dojindo Molecular Technologies, Japan) by following the manufacturer's instructions. Briefly, cells were harvested at the indicated timepoints and transferred to new 24-well cell culture plates. A CCK-8 mixture solution was added to each well and incubated for another $3 \mathrm{~h}$. Then $150 \mu \mathrm{l}$ solution was transferred to new 96-well plates, and shaked for $15 \mathrm{~min}$. The absorbance was measured at $450 \mathrm{~nm}$ using an enzyme-linked immunosorbent assay (ELISA) microplate reader. The final value reported for each sample is the mean of three independent replicates.

\section{Quantitative real-time PCR}

RAW 264.7 cells were cultured on untreated or fabricated Ti plates and stimulated with RANKL $\left(100 \mathrm{ng} \mathrm{ml}^{-1}\right)$ for 2 days, with unstimulated cells acting as negative control (no RANKL). Total RNA was extracted and reverse-transcribed by first-strand cDNA synthesis (Thermo Scientific, Rockford, IL, USA). Real-time PCR was performed with the ABI 7300 system. Target gene expression was normalized to the housekeeping gene $\beta$-actin. 


\section{Western blot}

RAW 264.7 cells were cultured on sterilized untreated, CS/HA or CS/HA/NBD fabricated Ti plates and stimulated with RANKL (100 $\mathrm{ng} \mathrm{ml} \mathrm{ml}^{-1}$ ) for $15-30 \mathrm{~min}$. After stimulation, cells were washed with PBS and immediately lysed in buffer containing $20 \mathrm{mM}$ Tris- $\mathrm{HCl}, 150 \mathrm{mM} \mathrm{NaCl}, 1 \%$ Triton X-100 and protease and phosphate inhibitors. Cell lysates (30-40 $\mathrm{mg}$ total protein) were separated by $10 \%$ sodium dodecyl sulphatepolyacrylamide gel electrophoresis (SDS-PAGE) and transferred onto a polyvinylidene difluoride membrane. After blocking with $5 \%$ skim milk, the membrane was probed with anti-P65, anti-phosphorylated P65 (p-P65), anti-I $\kappa \mathrm{B} \alpha$ or antiphosphorylated $\mathrm{I} \kappa \mathrm{B} \alpha(\mathrm{p}-\mathrm{I} \kappa \mathrm{B} \alpha)$ antibodies (Cell Signaling, Santa Cruz, CA, USA). The same membrane was stripped and reprobed for $\beta$-actin. Protein bands were visualized by ECL-Plus reagent (GE Healthcare, Piscataway, NJ, USA).

Nuclear extracts were prepared using NE-PER Nuclear and Cytoplasmic extraction reagents (Thermo Scientific, Rockford, IL, USA). The nuclear fractions were resolved by SDS-PAGE. Primary antibodies against P65, laminin A (Sigma-Aldrich, St Louis, MO, USA) and histone H3 (Cell Signaling, Santa Cruz, CA, USA) were used according to the manufacturer's directions. Protein bands were visualized by ECL-Plus reagent (GE Healthcare, Piscataway, NJ, USA). No signal was detected with the isotype-matched control.

\section{Immunofluorescence analysis}

RAW 264.7 cells were cultured on sterilized untreated, CS/HA or CS/HA/NBD fabricated Ti plates as described above and then stimulated with RANKL (100 $\mathrm{ng} \mathrm{ml}^{-1}$ ) in $\alpha$-MEM medium. After 15-30 min and 4 days, cells were fixed with $4 \%$ formaldehyde and then incubated with primary P65 and TRAP accordingly or isotype-matched control antibodies followed by a biotinylated secondary antibody. Fluorescent signals were detected using Alexa Fluor 546-conjugated streptavidin before cells were immunostained with F-actin and counterstained with DAPI. Images were acquired using a Nikon 90i fluorescent microscope and analyzed using NIS-Elements software (Nikon). No signal was detected with isotype-matched control.

\section{Animal surgery}

The animal study was approved by the Animal Ethics Committee of Zhejiang University, Hangzhou, China, in accordance with the "Principles of Laboratory Animal Care and Use in Research" (Ministry of Health, Beijing, China). All SpragueDawley female rats (3 months-old) were placed in quarantine for 1 week prior to surgery. Then all rats received bilateral ovariectomization (OVX). Bred for 3 months after OVX, they were randomly assigned to undergo the following implantation. $10 \%$ chloral hydrate $\left(3 \mathrm{mg} \mathrm{kg}^{-1}\right)$ was injected intramuscularly (IM). The rats were fixed in right position with the proximal tibia shaved and the skin disinfected. A $0.5 \mathrm{~cm}$ longitudinal incision was made to expose the tibial tuberosity, and bone defects $(2 \mathrm{~mm} \times 4 \mathrm{~mm})$ were created with an electrical drill. Untreated and fabricated implants were fitted into the defects. The incisions were then closed in layers. Penicillin $\left(3.5 \mathrm{mg} \mathrm{kg}^{-1}\right)$ was administered IM for 3 days. The rats were allowed to move freely 1 day after surgery. After one and twelve weeks, the rats $n=6$ in each group) were euthanized by chloral hydrate overdose, and all specimens for the following tests were harvested.

\section{Micro-CT analysis}

Specimens containing implants and surrounding bone tissue were extracted ( $n=6$ in each group) and fixed in $4 \%$ paraformaldehyde for $24 \mathrm{~h}$, then transferred to $70 \%$ alcohol and placed in a Micro-CT scanner (Skyscan1176, Bruker MicroCT N.V., Belgium). Images were collected at an X-ray source voltage of $80 \mathrm{kV}$ and beam current of $313 \mu \mathrm{A}$ using filtered bremsstrahlung radiation. The scanning angular rotation was set at $360^{\circ}$ and the angular increment at $0.40^{\circ}$. Specimens were constructed and evaluated using 3D analysis software (CTvol, MicroCT N.V., Belgium), through which the 3D micro-architectural properties around the implant area were determined. The bone volume percentage (BV/TV), trabecular number (Tb.N) and trabecular separation (Tb.Sp) were calculated using the Otsu method for threshold choice.

\section{Histology and histomorphometry}

Tibias were harvested one week post-surgery, fixed in $4 \%$ paraformaldehyde, and then decalcified in a 20\% EDTA solution. After the implants were extracted, the samples were dehydrated in ethanol, embedded in paraffin, and sliced to a thickness of $10 \mu \mathrm{m}$ to create slides with these sections. Osteoclasts were considered as TRAP $^{+}$multinucleated cells and were visualized within the bone tissue along the implant using a staining kit (387A-1KT, Sigma-Aldrich, America). All the TRAPpositive cells were counted by microscopy (Leica DM4000, Germany). ${ }^{\mathbf{4 8}}$

The retrieval procedure was also performed 12 weeks after implantation. The tibias containing the implants were fixed in $4 \%$ neutral-buffered formaldehyde, dehydrated and embedded in methyl methacrylate. Undecalcified cut and ground sections containing the central part of the implants were produced at a final thickness of $30 \mu \mathrm{m}$ using a Macro cutting and grinding system (Exakt 310 CP series, Exakt Apparatebau, Norderstedt, Germany). Sections were stained with toluidine blue. Light microscopy (BX51, Olympus, Japan) and a PC-based image analysis system (Image-Pro PlusR, Media Cybernetics, Silver Springs, MD, USA) were used for histometric analysis. The percentages of BIC and BA were determined as described above.

\section{Statistical analysis}

In vitro experiments were carried out three times to obtain mean and standard error from independent experiments. In vivo experiments were carried out with 6 animals per group. Statistical significance was determined by one-way ANOVA with Turkey's post hoc test for comparisons between multiple groups at the $P<0.05$ level. 


\section{Results and discussion}

\section{Characterization of NBD coating of Ti surfaces}

In our study, CS/HA and CS/HA/NBD were fabricated on roughed Ti plates and implants using the LBL technique separately as described previously. ${ }^{49}$ The isoelectric points of CS, HA and NBD are 7.1, 2.9 and 10.43, respectively. When dissolved in a solution at $\mathrm{pH} 5.0, \mathrm{CS}$ and NBD can carry a positive charge while HA carries a negative charge. ${ }^{45}$ Adsorption of positive and negative charges by these three biomaterials made their successful fabrication on the Ti surface using the LBL technology possible. When visualized on a lower-power field, SEM revealed a roughed structure for all three groups. In the CS/HAand CS/HA/NBD-coated groups, the roughness seemed smaller than in the untreated control group. Under a high-power field, SEM analysis revealed a rough surface morphology with clear valley-like structures on the untreated Ti plates. Meanwhile, trivial granular structures within the roughed structure were observed on the CS/HA- and CS/HA/NBD-coated Ti plates, resulting from the layers of biological films fabricated onto the Ti surface, and the NBD-coated group with larger molecular weight displayed more granular structures than the CS/HAcoated group (Fig. 1). AFM images displayed a similar rough surface morphology on the untreated Ti substrates. However, a relatively soft layer on top of the hard surface was observed on the CS/HA and CS/HA/NBD substrates (Fig. 1). We discovered the same rough structure on the surface of the untreated $\mathrm{Ti}$ implants, and the similar trivial granular structures on the CS/ HA- and CS/HA/NBD-coated implant surfaces (Fig. 2). Although slight difference of morphology was observed, quantification of surface roughness uncovered no significant differences between the groups (Fig. 3a). This confirms that the fabrication process did not change the surface morphology statistically. It was previously reported that the roughness of the Ti surface accelerates osteoclast differentiation. ${ }^{50}$ In our study, the NBD coating did not significantly change the surface roughness, suggesting the following results are due to the presence of NBD rather than differences in surface morphology between different groups.

The contact angle test was performed to investigate the wetability of different Ti surfaces (Fig. 3b). The mean standard \pm deviations of the contact angle on untreated, CS/HA-, and CS/ HA/NBD-coated Ti surfaces were $93.98 \pm 4.089,71.38 \pm 1.89$, and $33.42 \pm 2.06$, respectively $(p<0.05)$ (Fig. $3 c)$. The wetability of the multilayered $\mathrm{Ti}$ films was enhanced compared to native $\mathrm{Ti}$ surfaces, and NBD coating made the surface more hydrophilic than the CS/HA coating, suggesting the NBD coating increased

\section{Control (Ti)}

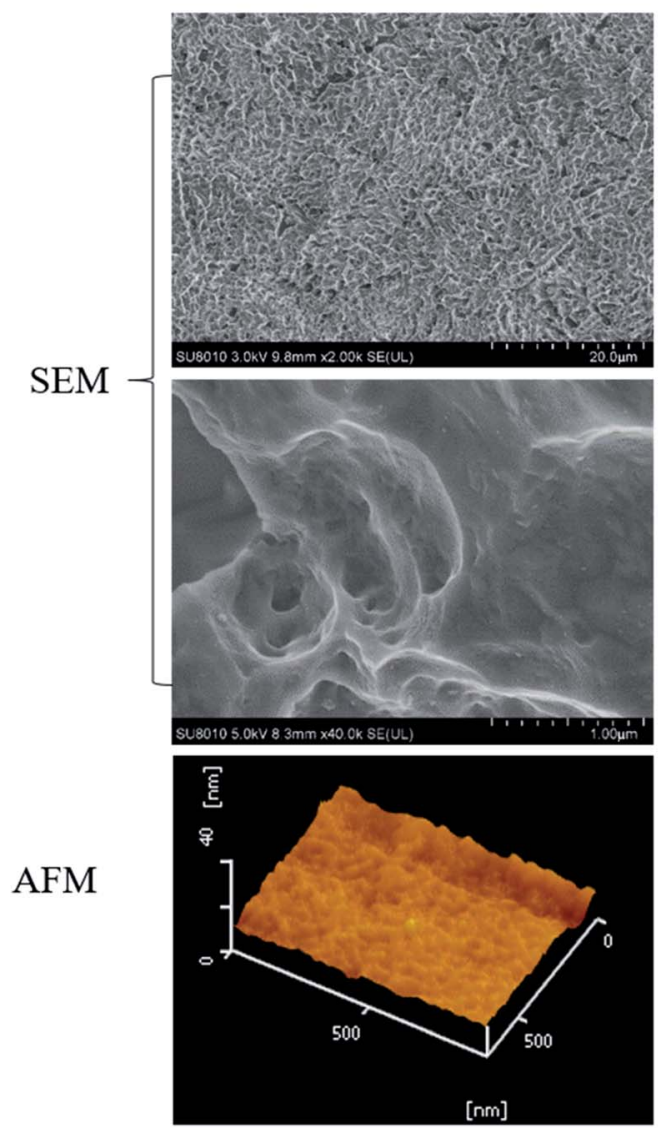

$\mathrm{CS} / \mathrm{HA}$
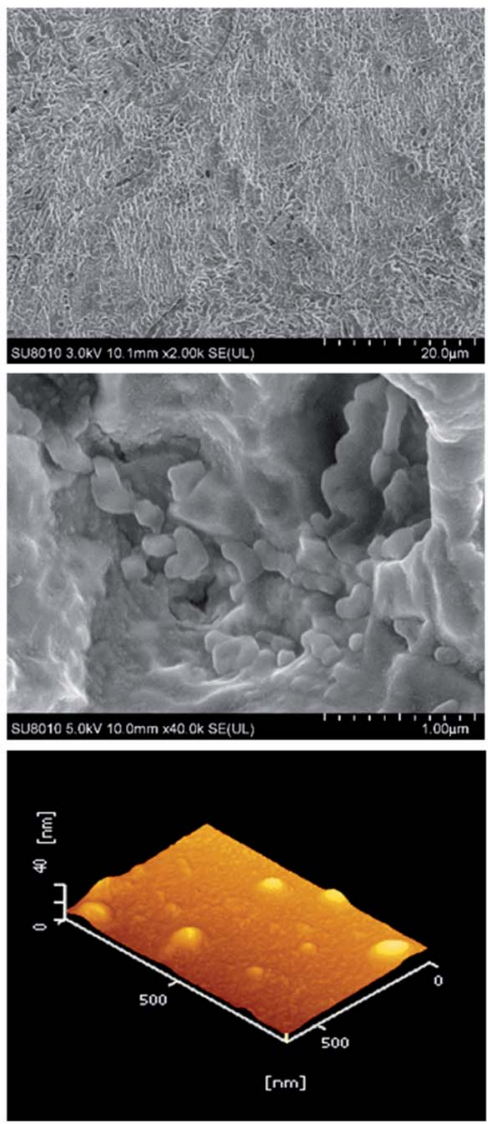

CS/HA/NBD
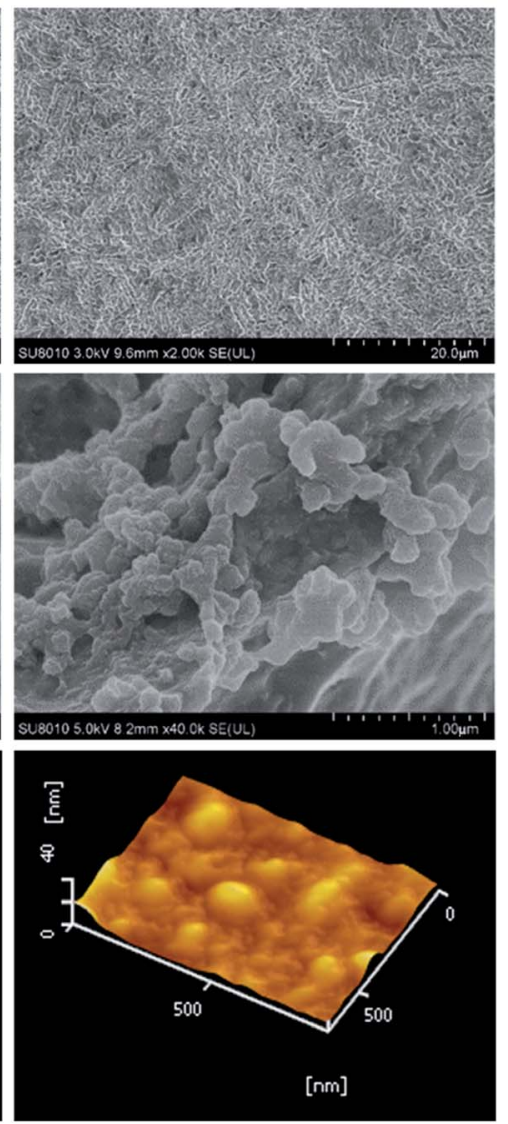

Fig. 1 Morphological characterization of surface of coated Ti plates. Assessment of surface morphology of untreated (control), CS/HA-, and CS/ HA/NBD-coated Ti plates using SEM and AFM. 


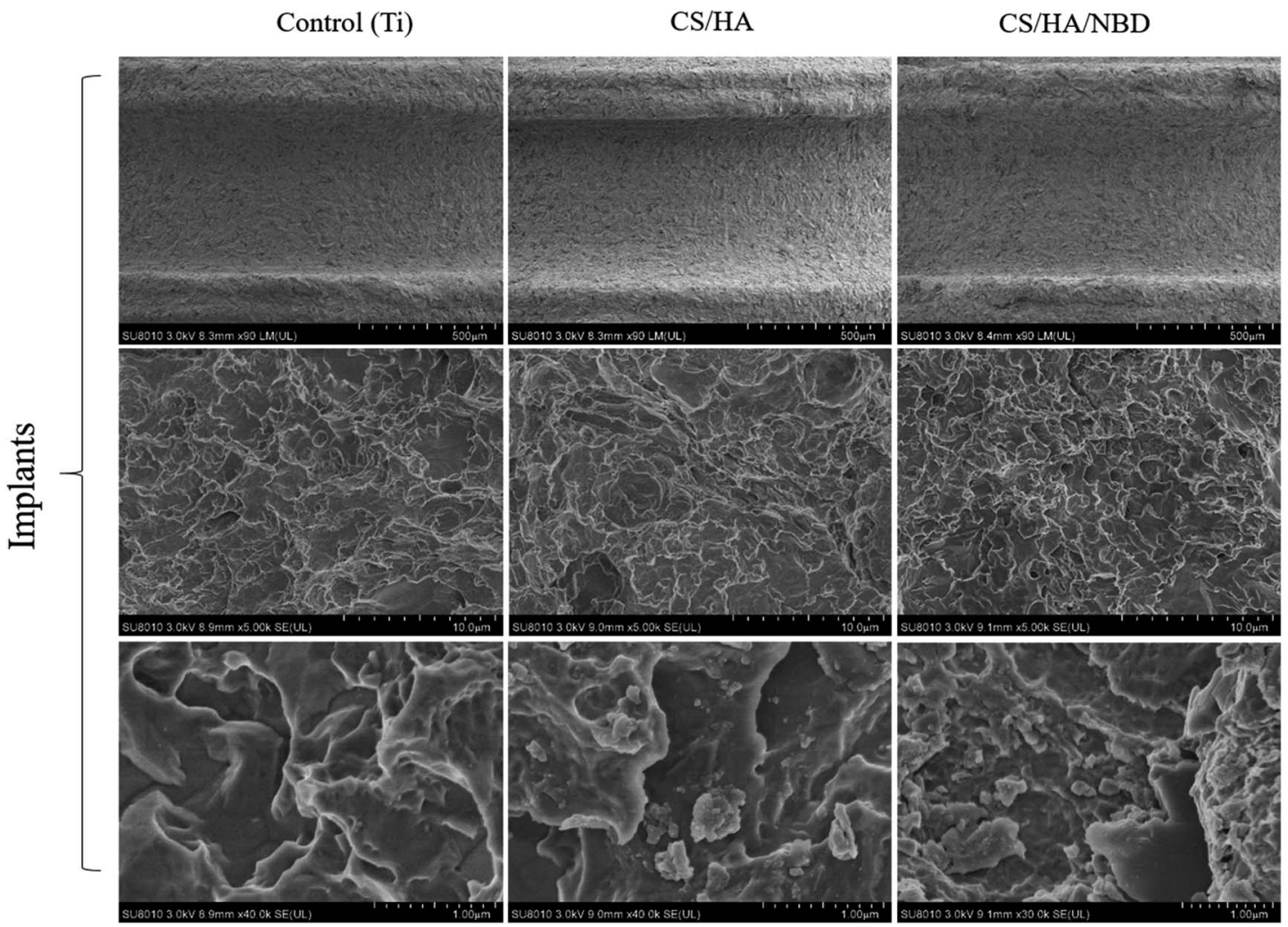

Fig. 2 Morphological characterization of coated Ti implants. Assessment of surface morphology of untreated (control), CS/HA-, and CS/HA/ NBD-coated Ti implants by SEM.

a

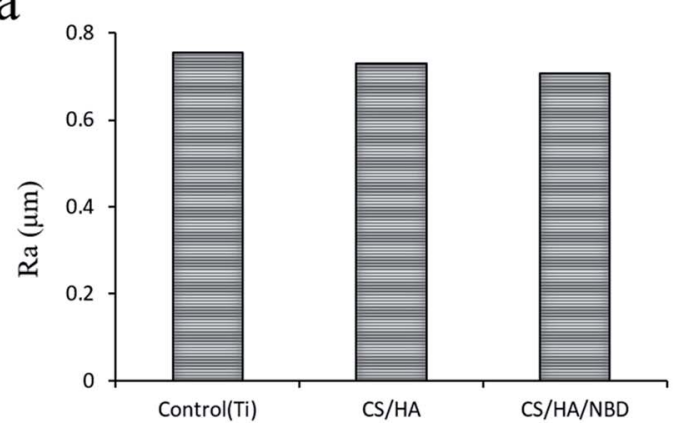

C

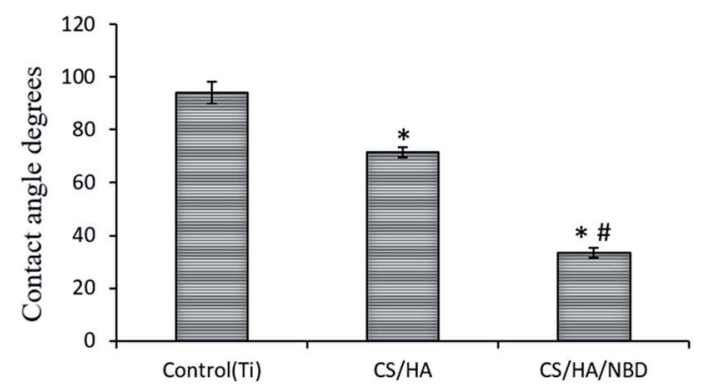

b

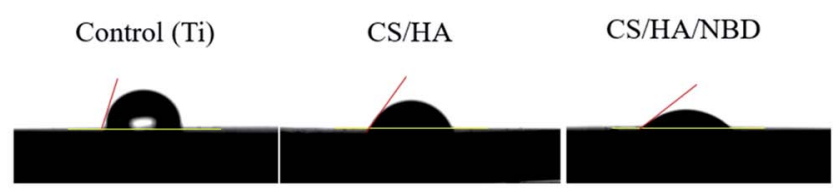

d

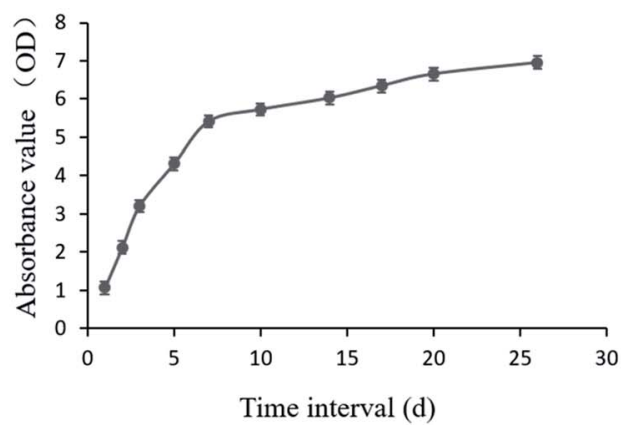

Fig. 3 (a) Analysis of roughness of untreated (control), CS/HA-, and CS/HA/NBD-coated Ti surfaces. (b and c) wetability of different Ti surfaces as evaluated by the contact angles test. (d) Release kinetics of NBD peptide at different degradation times. Each value represents the mean \pm SEM of three independent measurements. *Significantly different from untreated Ti group $(P<0.05)$. "Significantly different from CS/HA coated group $(P<0.05)$. 
the biological activity on the surface of the $\mathrm{Ti}$ and were successfully fabricated on the rough Ti plates and implants using the LBL technique. Next, studies were carried out testing NBD release from the coating. As shown in Fig. 3d, NBD release was quick from days one to seven, but then stayed at a relatively low and stable rate after that. This may suggest that NBD plays a major role during the first week and maintains its function post-implantation.

The chemical compositions of different $\mathrm{Ti}$ plate surfaces were determined by XPS analyses. The high resolution XPS spectrum of carbon indicated that NBD (Fig. 4a) fabrication resulted in the highest peaks at the binding energies of $288 \mathrm{eV}$, corresponding to $\mathrm{C}=\mathrm{O}$, and $400 \mathrm{eV}$, corresponding to $\mathrm{C}-\mathrm{NH}_{2}$ (Fig. 4b). ${ }^{51}$ Signals corresponding to $\mathrm{S}, \mathrm{Ti}$ and $\mathrm{N}$ were also detected (Fig. 4c-e). The S signal stems from the methionine in NBD (Fig. 4a, red circle) and was only detected on the surface of NBD-coated Ti plates but not on untreated pure or CS/HAcoated Ti plates (Fig. 4c). A reduced Ti signal indicated a decrease in the uncoated Ti area, corresponding to CS/HA and $\mathrm{CS} / \mathrm{HA} / \mathrm{NBD}$ coating, and NBD coating resulted in the weakest $\mathrm{Ti}$ signal (Fig. 4d). Increased $\mathrm{N}$ represented an increase in coating substrate on the Ti area, with NBD increasing the most (Fig. 4e). Successful grafting of CS/HA/NBD onto the Ti surface was indicated by an appearance of $\mathrm{S}$ signal, a larger increase in $\mathrm{N}$ signal and a decrease in Ti signal compared to untreated Ti and CS/HA-coated surfaces.

\section{NBD coating inhibits osteoclastogenesis on Ti plates and reduces the expression of target genes involved in osteoclast formation}

RAW 264.7 cells were stimulated with RANKL to induce osteoclastogenesis in the presence or absence of the NBD peptide which was proved by both F-actin and TRAP staining (Fig. 5a). SEM results showed that osteoclasts in control group had multinucleated giant cells and big actin rings, whereas these were rarely observed in NBD-treated group (Fig. 5a). The NBDtreated group displayed a $72.1 \%$ reduction in the number of osteoclasts formed compared to the control group $(p<0.05)$ (Fig. 5b).

RAW 264.7 cells were cultured on untreated, CS/HA and CS/ HA/NBD-coated Ti plates and stimulated with RANKL for 4 days to induce osteoclastogenesis. NBD coating reduced osteoclast formation by $\sim 72 \%$ compared to untreated Ti or CS/HA-coated surfaces $(P<0.05)$ (Fig. 5c and d). The characteristic cell morphology on all investigated surfaces is presented and visualized by F-actin immunostaining and SEM (Fig. 5c). Cells mainly differed in size as well as in the formation and size of actin rings. Osteoclasts on both untreated Ti and CS/HA-coated a

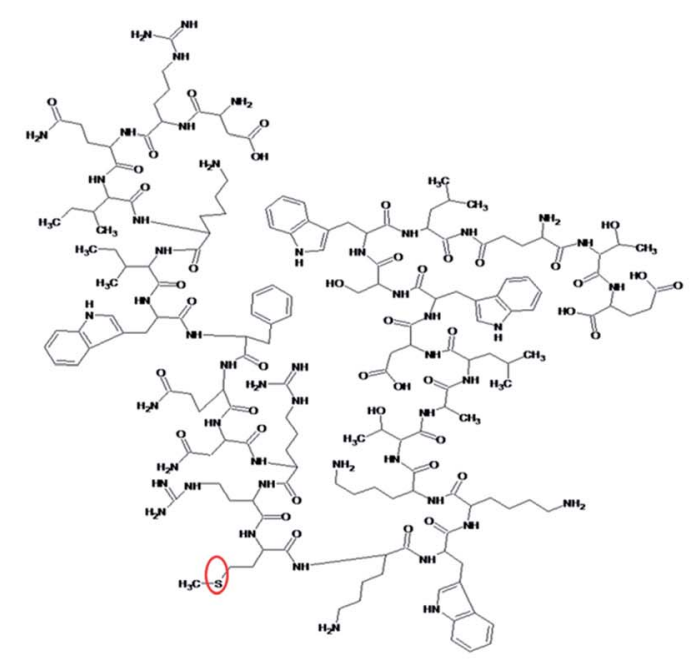

C

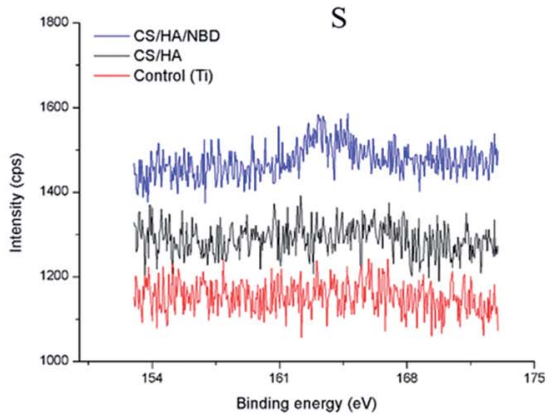

d b

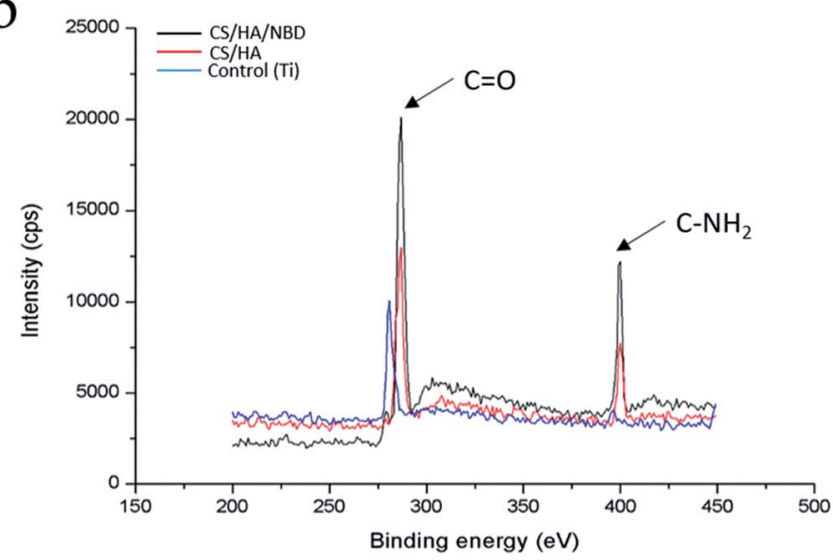

e

Fig. 4 Characterization of surface chemicals. (a) Chemical structure of NBD. (b-e) Chemical element analyses of different Ti surfaces by XPS spectra. (b) C 1s- and N 1s-related covalent bonding spectra, (c) S 2p spectra, (d) Ti 2p spectra, and (e) N 1s spectra. Red circle indicates S element from methionine in NBD. 


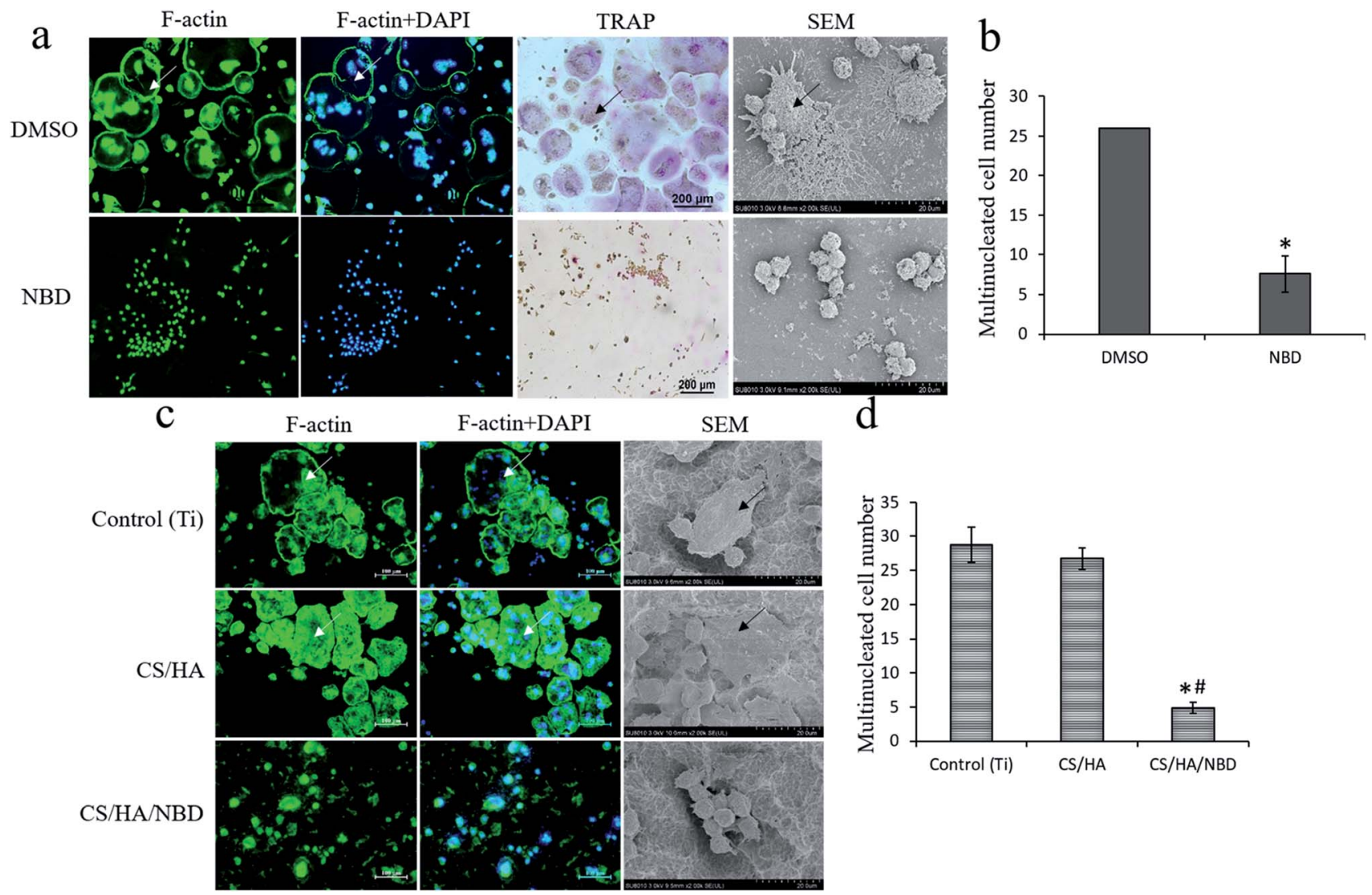

Fig. 5 NBD coating inhibits osteoclastogenesis on Ti plates. (a) Representative images of multinucleated osteoclasts by F-actin immunostaining, TRAP and SEM. White arrows indicate multinucleated cells in immunostaining images. Black arrows indicate multinucleated cells in TRAP and SEM images. (b) Quantification of multinucleated osteoclasts with more than 3 nuclei per osteoclasts. Each value represents the mean \pm SEM of three independent measurements. *Significantly different from DMSO-treated control group ( $p<0.05)$. (c) RAW 264.7 cells were cultured on untreated, CS/HA- or CS/HA/NBD-coated Ti plates and stimulated with RANKL $\left(100 \mathrm{ng} \mathrm{ml}^{-1}\right)$ for 4 days before cells were fixed and immunostained with F-actin and DAPI. The morphologies of multinucleated cells were also characterized by SEM. Multinucleated cells containing more than three nuclei were counted as osteoclasts. White arrows indicate multinucleated cells in immunostaining images. Black arrows indicate multinucleated cells in SEM images. (d) Quantification of multinucleated osteoclasts with more than 3 nuclei per osteoclasts. Each value represents the mean \pm SEM of three independent measurements. *Significantly different from untreated Ti group $(P<0.05)$. ${ }^{*}$ Significantly different from $\mathrm{CS} / \mathrm{HA}$ coated group $(P<0.05)$

surfaces had multinucleated giant cells and big actin rings, whereas both were rarely observed on NBD-fabricated Ti surfaces. This result confirmed that the NBD we synthesized plays a similar inhibitory role on osteoclastogenesis as has previously been reported. ${ }^{43,44}$

Fig. $6 \mathrm{a}$ and $\mathrm{b}$ show the proliferation of RAW 264.7 cells at day 1,3 and 5 of incubation with or without stimulation. No significant difference was found in cell proliferation between the indicated time points, which suggests that NBD has more of inhibitory effects on osteoclastogenesis rather than toxic effect.

Several potential target genes were examined at the mRNA level to investigate the role of NBD fabrication on osteoclastogenesis in vitro. RANKL treatment increased NFATc1 expression by $10-11$ folds on both untreated and CS/HA-coated Ti surfaces $(p<0.05)$ whereas NBD fabrication reduced the RANKL-induced NFATc1 expression by $\sim 47 \%$ compared to the two control groups $(p<0.05)$ (Fig. 7a). Similar to NFATc1, NBD fabrication decreased the induction of other osteoclast formation and activity factors by RANKL, including those of MMP9 by $\sim 42 \%$, integrin $\alpha \nu$ by $20-49 \%$ and integrin $\beta_{3}$ by $\sim 82 \%(p<0.05)$ (Fig. 7b-d), while no differences were detected between untreated Ti and the CS/HA fabricated group. NFATc1 is a master regulator of osteoclastogenesis capable of inducing osteoclast precursors to differentiate and fuse to form the SZ and then mature osteoclasts. Integrin $\alpha \mathrm{v} \beta_{3}$ plays a major role in the adhesion and spread of osteoclasts, mediating aspects of cellular organization and function. Thus, NBD may impact SZ formation and osteoclast spreading by inhibiting NFATc1 expression, which in turn regulates Integrin $\alpha \mathrm{v} \beta_{3} \cdot{ }^{16,52} \mathrm{NBD}$ also modulated MMP9 expression which is needed for osteoclasts and responsible for the proteolytic degradation of bone matrix proteins.

Osseointegration is accomplished by bone remodeling around the implant site, ${ }^{1}$ and since successful bone remodeling in dental implants requires the balanced activation of both osteoclasts and osteoblasts, reducing but not completely inhibiting osteoclastogenesis is quite important for osseointegration. It has been reported that the NBD peptide blocks only 
a

RAW264.7 cells

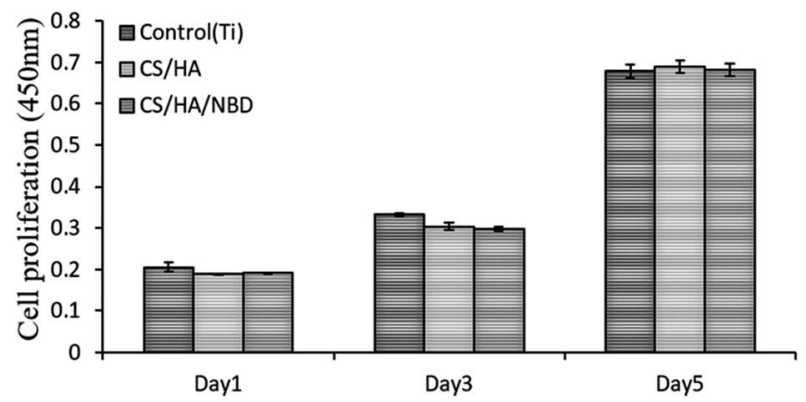

b

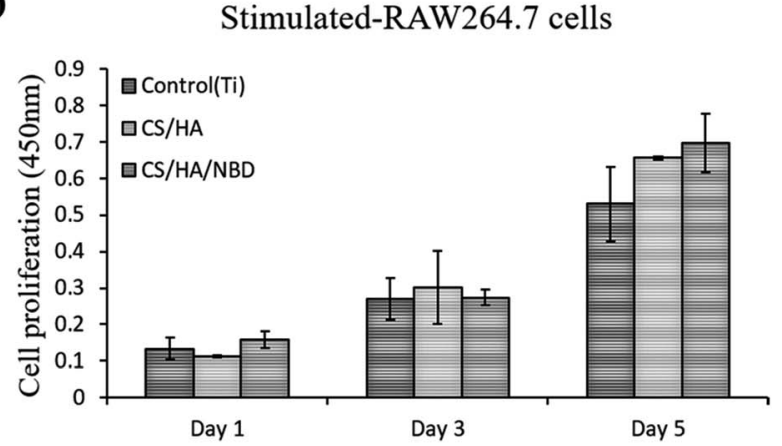

Fig. 6 Effect of NBD coating on cell proliferation. (a) Quantification of proliferation of RAW 264.7 cells without RANKL stimulation at day 1,3 and 5. (b) Quantification of proliferation of RANKL stimulated RAW 264.7 cells at day 1, 3 and 5 . Each value represents the mean \pm SEM of three independent measurements.

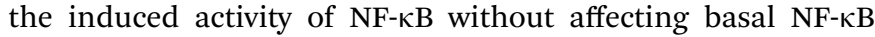
activity, ${ }^{53}$ which is in accordance with our observation that NBD coating partially but not completely inhibited osteoclastogenesis on Ti surfaces, which might be well-suited for the remodeling during implant osseointegration. Since the underlying mechanism by which NBD inhibits osteoclastogenesis had not been elucidated in previous studies, we demonstrated for the first time that NBD fabricated on Ti surfaces inhibits osteoclast formation and function by inhibiting NFATc1 which in turn regulates integrin $\alpha \mathrm{v} \beta_{3}$, which controls SZ formation, and MMP9, which is responsible for bone resorption.

Furthermore, we compared the number of osteoclast-like cells based on the formation of multinucleated cells in the different groups by measuring the activity of TRAP, a classic enzyme marker of osteoclasts, through immunofluorescence staining. Untreated $\mathrm{Ti}$ and $\mathrm{CS} / \mathrm{HA}-$ coated surfaces displayed much more robust TRAP expression and more multinucleated cells, which suggested that these two groups strongly stimulate osteoclastogenesis. In contrast, CS/HA/NBD-coated surfaces displayed fewer and smaller multinucleated cells and less TRAP expression, suggesting that NBD coating strongly inhibited osteoclastogenesis (Fig. 7e).

\section{NBD coating inhibits P65 and I $\kappa$ B $\alpha$ phosphorylation and P65 nuclear translocation}

The effects of NBD on P65 and I $\kappa \mathrm{B} \alpha$ phosphorylation and on P65 nuclear translocation in RAW 264.7 cells were assessed by western blot after 15-30 min of RANKL stimulation (Fig. 8a). NBD fabrication reduced ratio of $\mathrm{p}-\mathrm{I} \kappa \mathrm{B} \alpha$ to I $\mathrm{I} \mathrm{B} \alpha$ and p-P65 to P65. P65 protein accumulated in the nuclear fraction after 1530 min of RANKL stimulation in most cells in the untreated Ti

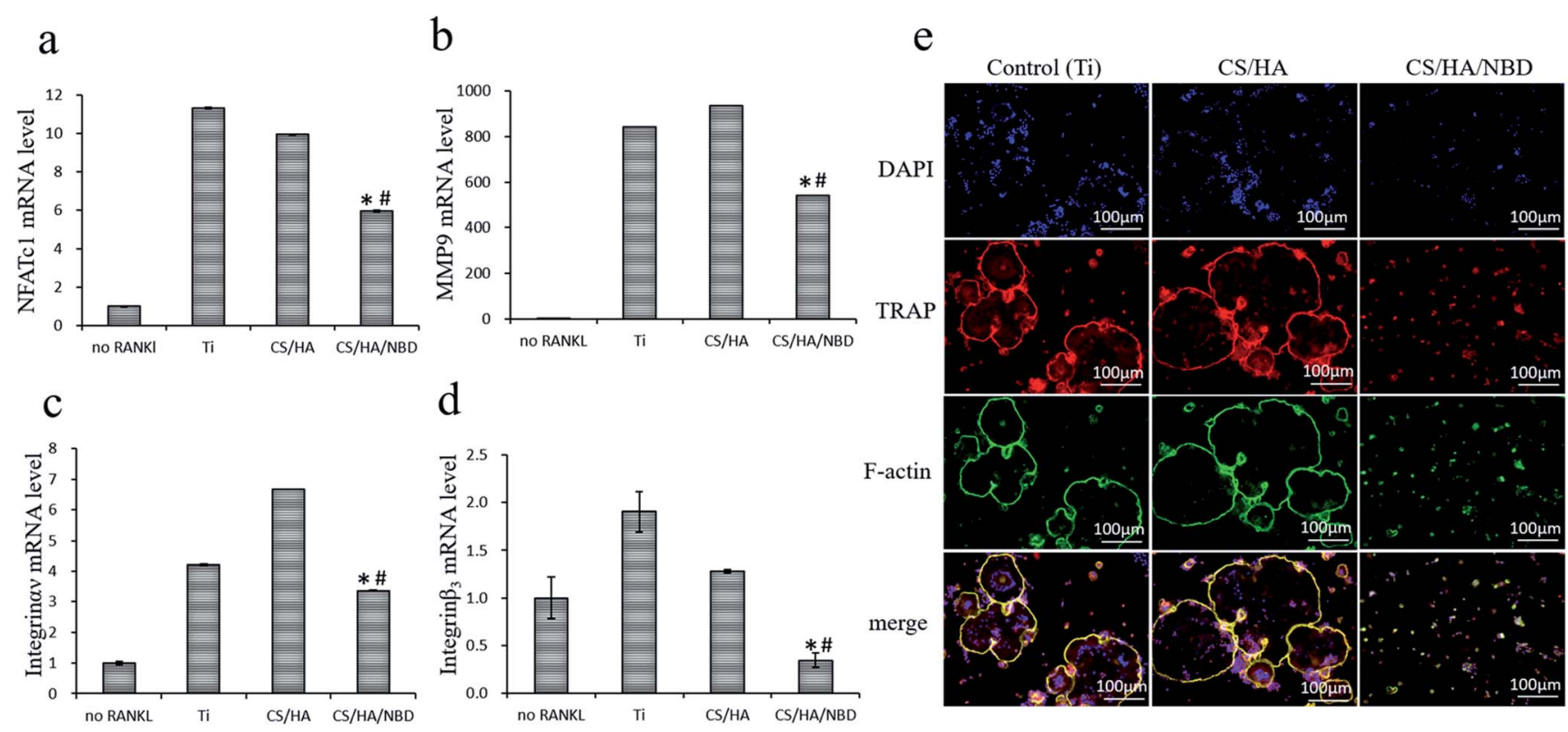

Fig. 7 NBD coating inhibits expression of target genes involved in osteoclast formation. Relative mRNA levels of (a) NFATc1, (b) MMP9, (c) integrin $\alpha v$ and (d) integrin $\beta_{3}$ compared to unstimulated control cells (no RANKL). Each value represents the mean \pm SEM of three independent measurements. *Significantly different from untreated Ti group $(P<0.05)$. " Significantly different from CS/HA coated group $(P<0.05)$. (e) Cells were cultured as described above for 4 days, fixed and immunostained for TRAP (red) and F-actin (green) and counterstained with DAPI (blue). No signal was detected with isotype-matched control antibodies (data not shown). 
and CS/HA fabricated groups, whereas in the NBD-coated group, P65 nuclear translocation were reduced.

Since P65 is active in the nucleus, its nuclear localization was also examined by immunofluorescence (Fig. 8b). P65 nuclear localization induced by RANKL could be detected after 1530 min of stimulation. In untreated and CS/HA-coated groups, P65 co-localized with DAPI in most cells while in the CS/HA/ NBD-coated group, P65 staining remained mostly outside the nucleus.

A crucial target of RANKL signaling is the activation of NF$\kappa \mathrm{B}$, which has been reported to play an important role in osteoclast differentiation. ${ }^{54}$ IKK induction requires recruitment of NEMO, which is essential for the phosphorylation of I $\mathrm{B}$ and subsequent activation of NF- $\kappa \mathrm{B} .{ }^{55}$ Shibata et al. ${ }^{56}$ reported that wtNBD peptide inhibited the phosphorylation and degradation of $\mathrm{I} \kappa \mathrm{B} \alpha$ by LPS treatment, which was quite similar to our results. In conclusion, we showed that NBD coated on Ti surfaces can reduced osteoclastogenesis by inhibiting P65 activation during the osteoclast formation process.

\section{NBD coating on the surface of roughed Ti implants inhibits} osteoclast formation at an early timepoint and enhances bone formation around implants in vivo

Osteoclast formation within the bone tissue around the implants was evaluated by TRAP staining at an early timepoint of one week post-implantation. The untreated and CS/HAcoated groups had more osteoclasts along the bone (Fig. 9a), as well as larger and more pseudopodia on the osteoclasts, than the NBD-coated group (Fig. 9b). Quantification revealed the number of osteoclasts in the NBD-coated group decreased by $\sim 33 \%$ compared to the untreated and CS/HA-groups $(p<0.05)$ (Fig. 9c). This suggests that the NBD coating on the implant surface prevents bone loss during the early stages of implantation to some extent, which may prevent bone loss overall and aid in sustaining more original bone tissue around the implant.

Based on the in vitro studies and the in vivo observations described above, pure Ti and NBD-coated implants were chosen for the following in vivo experiment. Osseointegration around implants was evaluated by Micro-CT analysis 12 weeks after implantation in OVX rats. Typical 3D images of implants and new bone were reconstructed. New bone ingrowth formation $500 \mu \mathrm{m}$ around implants was analyzed and compared to untreated Ti implants. NBD coating exhibited more favorable bone integration around the implants (Fig. 10a). Several parameters were examined, BV/TV and Tb.N were increased by $36.9 \%$ and $30.1 \%$, respectively, in the NBD-coated group compared with the control group $(p<0.05)$ (Fig. 10b and c) whereas Tb.Sp decreased by $29.4 \%$ ( $p<0.05$ ) (Fig. 10d). These structural assessments consistently demonstrated that the immobilized NBD used in this study enhanced pronounced bone-implant integration at a later timepoint.

Newly formed bone projecting directly from the margins of the bone defect toward the implants without signs of inflammation was observed in both untreated and CS/HA/NBD-coated groups. In the untreated group, there was limited new bone tissue attached to the implant with abundant connective tissue. The bone to implant contact was loosely organized within the

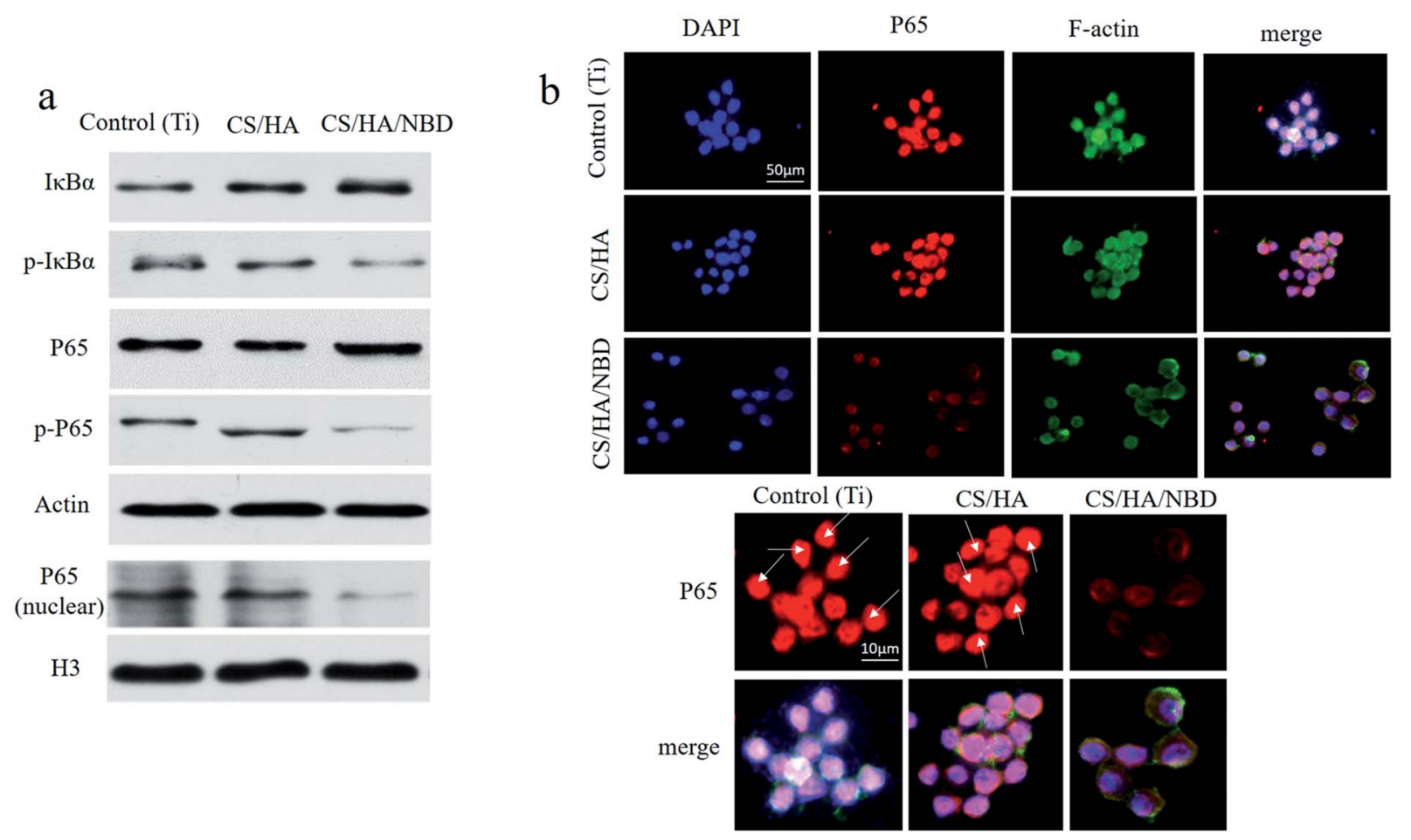

Fig. 8 NBD coating inhibits P65 and $I_{\kappa} B \alpha$ phosphorylation and P65 nuclear translocation. (a) Representative western blot images of $I_{\kappa} B \alpha, p-I_{\kappa} B \alpha$, P65, p-P65, and P65 nuclear translocation. (b) Cells were cultured as described above for 15-30 min, fixed and immunostained for P65 (red) and F-actin (green) and counterstained with DAPI (blue). Co-localization of P65 signal and DAPI nuclear stain indicated P65 nuclear localization. Lower panels with white arrows indicated P65 nuclear translocation. 
a

Control (Ti)
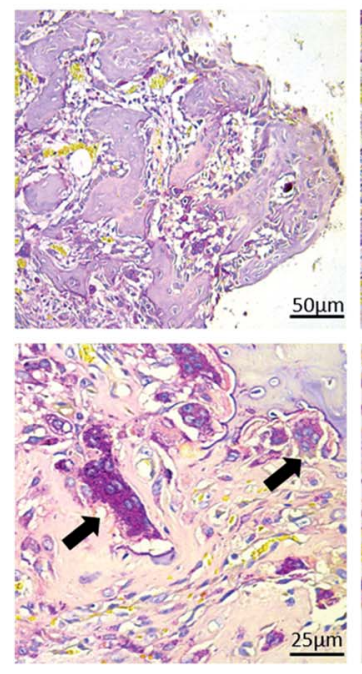

$\mathrm{CS} / \mathrm{HA}$
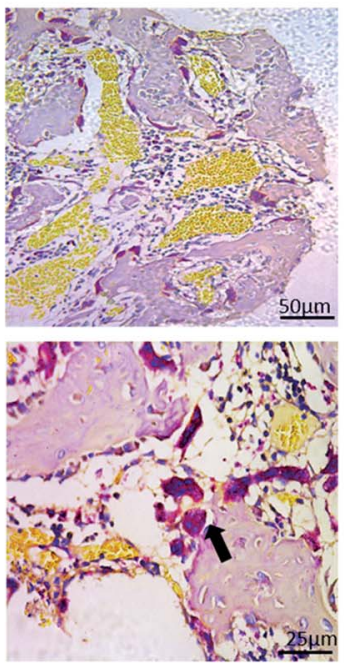

CS/HA/NBD
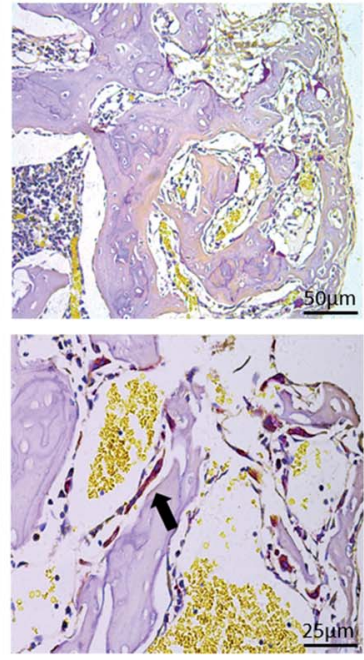

b Control (Ti) CS/HA CS/HA/NBD

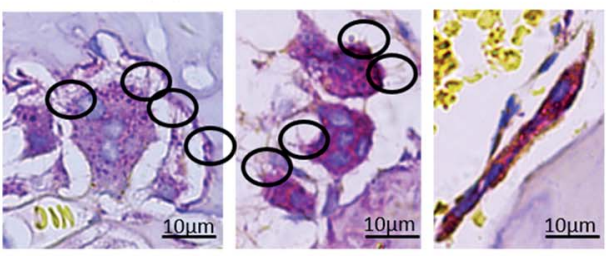

C

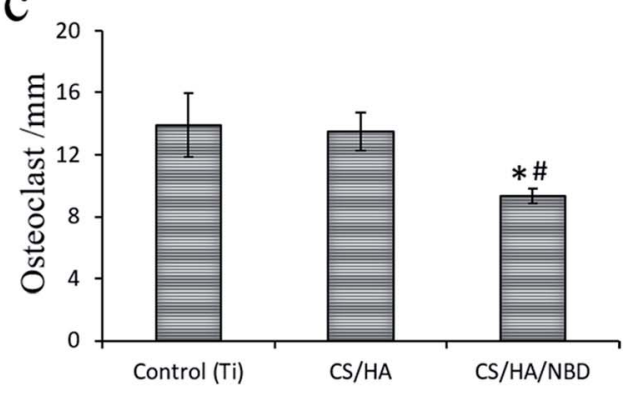

Fig. $9 \mathrm{CS} / \mathrm{HA} / \mathrm{NBD}$ coating inhibits osteoclast formation one week after implantation in vivo. (a) TRAP ${ }^{+}$osteoclasts lining the bone in the bone marrow cavity around the implant were stained with histostain and counted. Black arrow indicates multinucleated TRAP ${ }^{+}$cells. (b) Pseudopodia of osteoclasts were observed in untreated, CS/HA, and CS/HA/NBD cohorts. Black circle indicates pseudopodia of osteoclast. (c) Osteoclasts were counted as TRAP ${ }^{+}$multinucleated cells after TRAP staining. Each value is the mean \pm SEM for $n=6$ rats per group. *Significantly different from untreated Ti implant $(P<0.05)$. " Significantly different from CS/HA coated implants $(P<0.05)$.
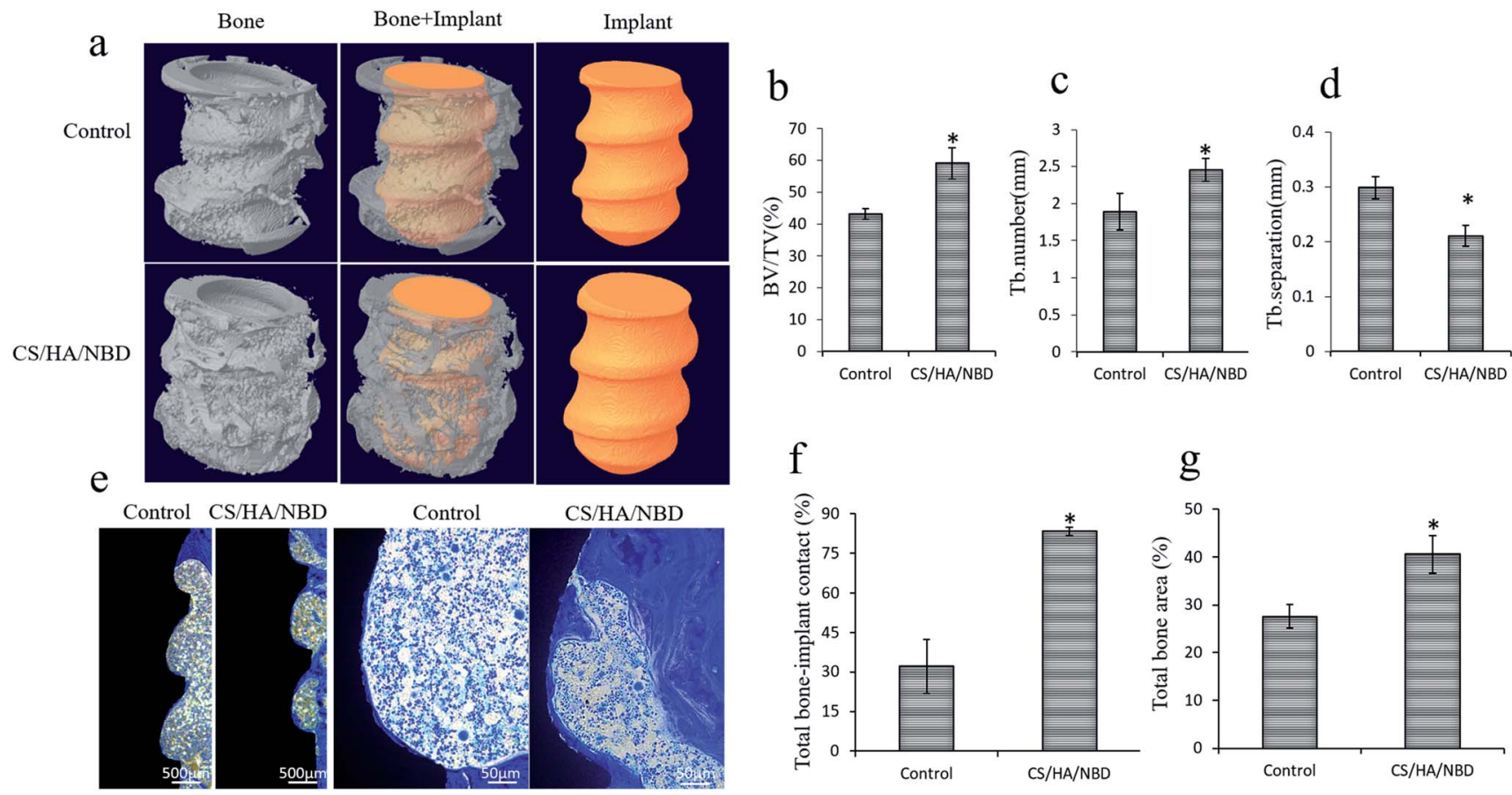

Fig. $10 \mathrm{CS} / \mathrm{HA} / \mathrm{NBD}$ coating of roughed Ti implant surfaces enhances osseointegration around implants. (a) Samples containing implants and surrounding bone tissues were extracted and fixed for Micro-CT analysis. Specimens were then constructed and evaluated with 3D analysis software. (b-d) Bone volume percentage (BV/TV), trabecular number (Tb.N) and trabecular separation (Tb.Sp). (e) Representative light micrographs of undecalcified toluidine blue-stained sections and histomorphometry of the bone interfaces to pure and NBD fabricated implants. ( $f$ ) Bone-implant contact $(\mathrm{BIC} \%)$ and $(\mathrm{g})$ bone area $(\mathrm{BA} \%)$ percentages. Each value is the mean $\pm \mathrm{SEM}$ for $n=6$ rats per group. *Significantly different from untreated Ti implant $(P<0.05)$.

implant. In the CS/HA/NBD-coated group, more bone growth was observed along the implant surface (Fig. 10e). Histomorphometric analysis revealed that the BIC increased 2.6 fold $(p<0.05)$ (Fig. 10f) and the BA 1.47 fold $(p<0.05)$ (Fig. 10g) in the NBD-coated group compared with the untreated Ti implant group. 
Implant-bone integration depends on bone remodeling and thus relies on both osteoclast and osteoblast activity, and in this study, our results suggest that NBD coated on Ti surfaces only partially inhibited osteoclast formation and function. Based on previous study, Li et al. ${ }^{57}$ demonstrated that NBD peptide can promote osteoblast differentiation when impaired by TNF- $\alpha$, suggesting that NBD may have an osteoblast-promoting role under inflammatory conditions. When inserted into the bone, implants will induce an inflammatory reaction, NBD may play dual roles by both reducing osteoclastogenesis and promoting osteoblast formation. Additional work is needed to assess this hypothesis.

\section{Conclusion}

This study strongly supports the hypothesis that NBD fabrication on the surface of $\mathrm{Ti}$ plates and implants exerts inhibitory effects on osteoclast formation and function in vitro by restraining the expression of NFATc1, which in turn regulates integrin $\alpha v \beta_{3}$ and MMP9 through the P65 signaling pathway, and synergistically enhances osseointegration around implants in vivo in OVX rat model. Compared to previously reported data, our approach may be more effective when applied to osteoporotic bone than to normal bone. Altogether, these findings hold great promise for the in vivo use of immobilized NBD peptides and selective inhibition of NF- $\mathrm{B}$ activation offers an effective therapeutic approach to select more suitable Ti-implant surface modification for enhancing implant osseointegration.

\section{Conflicts of interest}

There are no conflicts of interest to declare.

\section{Acknowledgements}

This work was supported by the National Natural Science Foundation of China (Grant No. 81771118, 81272157, 81600909). This work was also supported by Zhejiang Provincial Medical Science and Technology Project of China (No. 2018277520, 2015KYA146 and 2017RC009). We would like to thank Pinger Wang from Zhejiang Institute of Orthopedics and Traumatology for generously providing the Micro-CT and technological help. This manuscript has been thoroughly edited by a native English speaker from an editing company.

\section{Note and references}

1 I. Ericsson, C. B. Johansson, H. Bystedt and M. R. Norton, Clinical Oral Implants Research, 1994, 5, 202-206.

2 A. Palmquist, O. M. Omar, M. Esposito, J. Lausmaa and P. Thomsen, J. R. Soc., Interface, 2010, 7(suppl. 5), S515-S527.

3 S. M. Bang, H. J. Moon, Y. D. Kwon, J. Y. Yoo, A. Pae and I. K. Kwon, Clinical Oral Implants Research, 2014, 25, 831837.

4 E. S. Hartmann, M. I. Kohler, F. Huber, J. I. Redeker, B. Schmitt, M. Schmitt-Sody, B. Summer, A. Fottner,
V. Jansson and S. Mayer-Wagner, J. Orthop. Res., 2017, 35, 248-257.

5 G. G. Lugero, V. de Falco Caparbo, M. L. Guzzo, B. Konig Jr and V. Jorgetti, Implant Dentistry, 2000, 9, 303-309.

6 K. Gulati, M. Prideaux, M. Kogawa, L. Lima-Marques, G. J. Atkins, D. M. Findlay and D. Losic, J. Tissue Eng. Regener. Med., 2017, 11, 3313-3325.

7 F. Ozdal-Kurt, I. Tuglu, H. S. Vatansever, S. Tong, B. H. Sen and S. I. Deliloglu-Gurhan, Biotech. Histochem., 2016, 91, 412-422.

8 S. V. Reddy and G. D. Roodman, Crit. Rev. Eukaryotic Gene Expression, 1998, 8, 1-17.

9 N. Lampiasi, R. Russo and F. Zito, BioMed Res. Int., 2016, 2016, 9089610.

10 S. K. Verma, E. Leikina, K. Melikov and L. V. Chernomordik, Biochem. J., 2014, 464, 293-300.

11 D. L. Lacey, E. Timms, H. L. Tan, M. J. Kelley, C. R. Dunstan, T. Burgess, R. Elliott, A. Colombero, G. Elliott, S. Scully, H. Hsu, J. Sullivan, N. Hawkins, E. Davy, C. Capparelli, A. Eli, Y. X. Qian, S. Kaufman, I. Sarosi, V. Shalhoub, G. Senaldi, J. Guo, J. Delaney and W. J. Boyle, Cell, 1998, 93, 165-176.

12 W. J. Boyle, W. S. Simonet and D. L. Lacey, Nature, 2003, 423, 337-342.

13 M. Asagiri, K. Sato, T. Usami, S. Ochi, H. Nishina, H. Yoshida, I. Morita, E. F. Wagner, T. W. Mak, E. Serfling and H. Takayanagi, J. Exp. Med., 2005, 202, 1261-1269.

14 Y. H. Chiu, E. Schwarz, D. Li, Y. Xu, T. R. Sheu, J. Li, K. L. Bentley, C. Feng, B. Wang, J. C. Wang, L. AlbertorioSaez, R. Wood, M. Kim, W. Wang and C. T. Ritchlin, J. Cell. Physiol., 2017, 232, 2538-2549.

15 A. W. Lange and K. E. Yutzey, Dev. Biol., 2006, 292, 407-417. 16 T. N. Crotti, S. M. Sharma, J. D. Fleming, M. R. Flannery, M. C. Ostrowski, S. R. Goldring and K. P. McHugh, J. Cell. Physiol., 2008, 215, 636-644.

17 I. Song, J. H. Kim, K. Kim, H. M. Jin, B. U. Youn and N. Kim, FEBS Lett., 2009, 583, 2435-2440.

18 M. A. Horton, M. L. Taylor, T. R. Arnett and M. H. Helfrich, Exp. Cell Res., 1991, 195, 368-375.

19 V. W. Engleman, G. A. Nickols, F. P. Ross, M. A. Horton, D. W. Griggs, S. L. Settle, P. G. Ruminski and S. L. Teitelbaum, J. Clin. Invest., 1997, 99, 2284-2292.

20 O. Destaing, F. Saltel, J. C. Geminard, P. Jurdic and F. Bard, Mol. Biol. Cell, 2003, 14, 407-416.

21 P. Jurdic, F. Saltel, A. Chabadel and O. Destaing, Eur. J. Cell Biol., 2006, 85, 195-202.

22 H. K. Vaananen, E. K. Karhukorpi, K. Sundquist, B. Wallmark, I. Roininen, T. Hentunen, J. Tuukkanen and P. Lakkakorpi, J. Cell Biol., 1990, 111, 1305-1311.

23 M. Kogawa, K. Hisatake, G. J. Atkins, D. M. Findlay, Y. Enoki, T. Sato, P. C. Gray, Y. Kanesaki-Yatsuka, P. H. Anderson, S. Wada, N. Kato, A. Fukuda, S. Katayama, M. Tsujimoto, T. Yoda, T. Suda, Y. Okazaki and M. Matsumoto, J. Biol. Chem., 2013, 288, 31299-31312.

24 S. Vaira, M. Alhawagri, I. Anwisye, H. Kitaura, R. Faccio and D. V. Novack, J. Clin. Invest., 2008, 118, 2088-2097. 
25 N. S. Soysa and N. Alles, Biochem. Biophys. Res. Commun., 2009, 378, 1-5.

26 Y. Xia, S. Shen and I. M. Verma, Cancer Immunol. Res., 2014, 2, 823-830.

27 P. Geng, J. Ou, J. Li, Y. Liao, N. Wang, R. Sa, L. Xiang and H. Liang, Medicine, 2015, 94, e1024.

28 Y. Abu-Amer, Osteoporosis Int., 2013, 24, 2377-2386.

29 X. Li, P. E. Massa, A. Hanidu, G. W. Peet, P. Aro, A. Savitt, S. Mische, J. Li and K. B. Marcu, J. Biol. Chem., 2002, 277, 45129-45140.

30 J. Ye, X. Xie, L. Tarassishin and M. S. Horwitz, J. Biol. Chem., 2000, 275, 9882-9889.

31 A. Desai, N. Singh and R. Raghubir, Neurochem. Int., 2010, 57, 876-883.

32 N. S. Soysa, N. Alles, H. Shimokawa, E. Jimi, K. Aoki and K. Ohya, J. Bone Miner. Metab., 2009, 27, 131-139.

33 N. Alles, N. S. Soysa, J. Hayashi, M. Khan, A. Shimoda, H. Shimokawa, O. Ritzeler, K. Akiyoshi, K. Aoki and K. Ohya, Endocrinology, 2010, 151, 4626-4634.

34 I. Strickland and S. Ghosh, Ann. Rheum. Dis., 2006, 65(suppl. 3), iii75-iii82.

35 H. S. Alghamdi and J. A. Jansen, Tissue Eng., Part B, 2013, 19, 233-253.

36 S. P. Johnsen, H. T. Sorensen, U. Lucht, K. Soballe, S. Overgaard and A. B. Pedersen, J. Bone Jt. Surg., Br. Vol., 2006, 88, 1303-1308.

37 H. Li, J. Jiang, Y. Ge, J. Xu, P. Zhang, W. Zhong and S. Chen, J. Biomater. Sci., Polym. Ed., 2013, 24, 431-446.

38 H. Lu and N. Hu, J. Phys. Chem. B, 2006, 110, 23710-23718.

39 H. Li, Y. Ge, P. Zhang, L. Wu and S. Chen, J. Biomater. Sci., Polym. Ed., 2012, 23, 425-438.

40 Y. Huang, Q. Luo, X. Li, F. Zhang and S. Zhao, Acta Biomater., 2012, 8, 866-877.

41 J. Shi, L. L. Dong, F. He, S. Zhao and G. L. Yang, Oral Surg. Oral Med. Oral Pathol. Oral Radiol., 2013, 116, e311-316.
42 X. Li, et al., Polym. Adv. Technol., 2012, 23, 756-764.

43 E. Jimi, K. Aoki, H. Saito, F. D'Acquisto, M. J. May, I. Nakamura, T. Sudo, T. Kojima, F. Okamoto, H. Fukushima, K. Okabe, K. Ohya and S. Ghosh, Nat. Med., 2004, 10, 617-624.

44 S. Dai, T. Hirayama, S. Abbas and Y. Abu-Amer, J. Biol. Chem., 2004, 279, 37219-37222.

45 J. Shi, Y. Liu, Y. Wang, J. Zhang, S. Zhao and G. Yang, Sci. Rep., 2015, 5, 16336.

46 Y. P. Fan, X. Y. Chen, Y. Chen, G. L. Yang, H. M. Wang and F. M. He, Clinical Oral Implants Research, 2017, 28, 911-919. 47 Y. Wang, G. Dong, H. H. Jeon, M. Elazizi, L. B. La, A. Hameedaldeen, E. Xiao, C. Tian, S. Alsadun, Y. Choi and D. T. Graves, J. Immunol., 2015, 194, 2878-2887.

48 M. S. Lee, H. S. Kim, J. T. Yeon, S. W. Choi, C. H. Chun, H. B. Kwak and J. Oh, J. Immunol., 2009, 183, 3390-3399.

49 G. Decher, Science, 1997, 277, 1232-1237.

50 S. Makihira, Y. Mine, E. Kosaka and H. Nikawa, Dent. Mater. J., 2007, 26, 739-745.

51 B. Nie, H. Ao, J. Zhou, T. Tang and B. Yue, Colloids Surf., B, 2016, 145, 728-739.

52 T. N. Crotti, M. Flannery, N. C. Walsh, J. D. Fleming, S. R. Goldring and K. P. McHugh, Gene, 2006, 372, 92-102.

53 E. Jimi and S. Ghosh, Immunol. Rev., 2005, 208, 80-87.

54 L. Xing, T. P. Bushnell, L. Carlson, Z. Tai, M. Tondravi, U. Siebenlist, F. Young and B. F. Boyce, J. Bone Miner. Res., 2002, 17, 1200-1210.

55 M. J. May, F. D'Acquisto, L. A. Madge, J. Glockner, J. S. Pober and S. Ghosh, Science, 2000, 289, 1550-1554.

56 W. Shibata, S. Maeda, Y. Hikiba, A. Yanai, T. Ohmae, K. Sakamoto, H. Nakagawa, K. Ogura and M. Omata, J. Immunol., 2007, 179, 2681-2685.

57 W. Li, B. Yu, M. Li, D. Sun, Y. Hu, M. Zhao, C. B. Cui and S. Hou, Biochem. Biophys. Res. Commun., 2010, 391, 12281233. 